\title{
PAPER
}

Cite this: Metallomics, 2013 5, 1247

Received 28th February 2013, Accepted 5th April 2013

DOI: $10.1039 / \mathrm{c} 3 \mathrm{mt} 00060 \mathrm{e}$

www.rsc.org/metallomics

\section{Iron distribution through the developmental stages of Medicago truncatula nodules $\dagger$}

\author{
Benjamín Rodríguez-Haas, ${ }^{a}$ Lydia Finney, ${ }^{\mathrm{b}}$ Stefan Vogt, ${ }^{\mathrm{b}}$ Pablo González-Melendi, ${ }^{\mathrm{a}}$ \\ Juan Imperial ${ }^{\mathrm{ac}}$ and Manuel González-Guerrero*a
}

\begin{abstract}
Paramount to symbiotic nitrogen fixation (SNF) is the synthesis of a number of metalloenzymes that use iron as a critical component of their catalytical core. Since this process is carried out by endosymbiotic rhizobia living in legume root nodules, the mechanisms involved in iron delivery to the rhizobia-containing cells are critical for SNF. In order to gain insight into iron transport to the nodule, we have used synchrotron-based X-ray fluorescence to determine the spatio-temporal distribution of this metal in nodules of the legume Medicago truncatula with hitherto unattained sensitivity and resolution. The data support a model in which iron is released from the vasculature into the apoplast of the infection/differentiation zone of the nodule (zone II). The infected cell subsequently takes up this apoplastic iron and delivers it to the symbiosome and the secretory system to synthesize ferroproteins. Upon senescence, iron is relocated to the vasculature to be reused by the shoot. These observations highlight the important role of yet to be discovered metal transporters in iron compartmentalization in the nodule and in the recovery of an essential and scarce nutrient for flowering and seed production.
\end{abstract}

\section{Introduction}

Symbiotic nitrogen fixation (SNF) plays a major role in the nitrogen cycle in natural terrestrial ecosystems. ${ }^{1}$ In its most common form, it is carried out by the Rhizobium-legume symbiosis in a differentiated root structure, the nodule. ${ }^{2}$ The symbiosis is established upon exchange of chemical signals that trigger the curling of a radical hair, surrounding locally-accumulated rhizobia, from which an infection thread will develop to guide invasive rhizobia to the nodule cortex. ${ }^{3}$ Rhizobia are released in the cytosol of the host cell by an endocytic-like process. ${ }^{4}$ Subsequently, the endocytic rhizobia differentiate into bacteroids and together with the "endocytic" vesicle constitute an organelle-like structure, the symbiosome. ${ }^{5}$ In the microaerobic environment achieved within the nodule, bacteroids produce the enzyme nitrogenase, which is directly responsible for nitrogen fixation. ${ }^{6}$

There are two main developmental types of nodule: determinate and indeterminate. ${ }^{7}$ Their main difference is that the

\footnotetext{
${ }^{a}$ Centro de Biotecnología y Genómica de Plantas (CBGP), Universidad Politécnica de Madrid, Campus de Montegancedo, Crta. M40 km 37, 28223 Pozuelo de Alarcón, Madrid, Spain. E-mail: manuel.gonzalez@upm.es; Tel: +34 913364568

${ }^{b}$ X-Ray Science Division, Advanced Photon Source, Argonne National Laboratory, Argonne, Illinois, USA

${ }^{c}$ Consejo Superior de Investigaciones Cientificas (CSIC), Madrid, Spain

$\dagger$ Electronic supplementary information (ESI) available. See DOI: 10.1039/ c3mt00060e
}

indeterminate type, developed by Medicago and Vicia plants among other plants, has an apical meristem that allows nodule growth as new rhizobia are being released right after the meristematic region. As a consequence, temporal development of the symbiosis can be studied by observing spatial differentiation along an indeterminate nodule. Four zones have been defined in a mature indeterminate nodule (Fig. 1): zone $\mathrm{I}$ is the apical meristem, zone II is where rhizobia are released into the host cells and differentiate, zone III is where nitrogen fixation occurs in a microaerobic environment, and zone IV is the senescent zone. ${ }^{5}$

Iron plays an essential part in SNF. ${ }^{8}$ It is the cofactor of the main enzymes involved in this process, from leghemoglobin, protein responsible for the steady supply of low levels of oxygen in the microaerobic environment in which the reaction takes place, ${ }^{9}$ to nitrogenase, the enzyme that catalyzes the reduction of $\mathrm{N}_{2}$ into two $\mathrm{NH}_{4}{ }^{+10} \cdot{ }^{10}$ These and other nodule ferroproteins are expressed at very high levels, to the extent that they induce the iron deficiency response in the host legume. ${ }^{11}$ Consequently, the mechanisms involved in iron delivery to the symbiosome are critical for SNF.

However, very little is known about metal trafficking to the symbiosome. Previous studies by Moreau, Kaiser and collaborators identified two soybean symbiosome-specific metal transporters, GmZIP1 and GmDMT1. ${ }^{12}$ GmDMT1 is an Nramp family member putatively involved in $\mathrm{Fe}^{2+}$ transport. Its function has been 


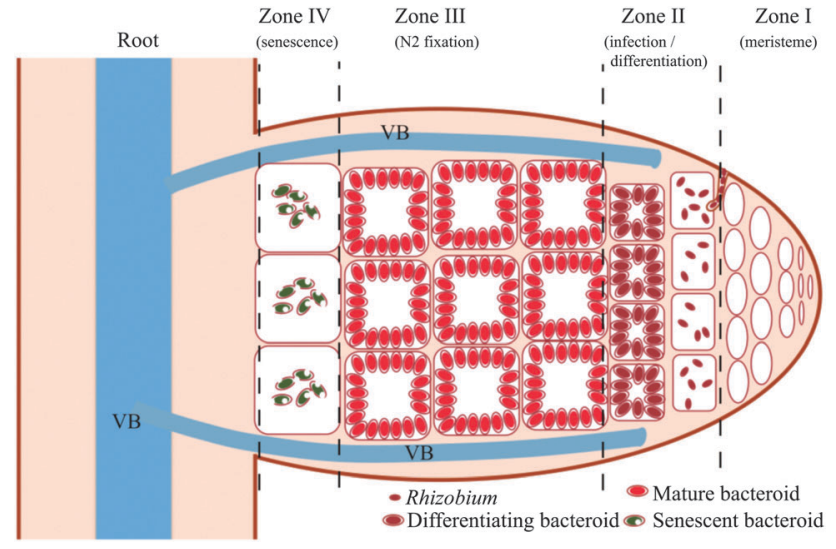

Fig. 1 Diagram of indeterminate nodule development. The nodule grows by meristematic cell divisions in zone I. In zone II, rhizobia are released by the infection thread into cortical cells by an endocytic process. There, rhizobia differentiate into bacteroids that in zone III synthesize nitrogenase and other enzymes involved in nitrogen fixation. After some weeks, the nodule starts senescing and bacteroids are degraded (zone IV). VB indicates Vascular Bundle.

suggested to be that of iron transport into the symbiosome, although that would be the direction of transport opposite to that of already characterized Nramp proteins. ${ }^{13}$ More recently, a legume VIT1 homologue, SEN1 has been connected to iron transport in the nodule. ${ }^{14}$ Although no detailed information about its subcellular localization was provided, SEN1 is somehow involved in providing iron to nitrogenase, since its mutation results in $f i x^{-}$nodules. ${ }^{14}$ Similar observations also support a role of citrate and citrate transporters in iron delivery to the nodule. ${ }^{15}$

To ascertain how metal transporters coordinate metal delivery for SNF, the origin of the iron delivered must be first found out. Theoretically, three possible routes could be followed: (i) pre-existing iron stores formed in the nodule primordium, (ii) increased epidermal uptake in the nodule surface, and (iii) increased delivery through the vasculature. Elucidating which of these three is the most important is relevant towards determining which transporters might mediate this process and understanding how this very scarce oligonutrient is managed in legumes.

Synchrotron-based X-ray fluorescence (S-XRF) was used to evaluate the likelihood of each path by determining metal distribution in the different developmental zones in the nodule. This is a powerful technique that has provided the explanation for a number of metal-dependent phenotypes. ${ }^{16}$ Its resolution allows us to study submicromolar concentrations of metals with subcellular resolution. ${ }^{16 a, c}$ If the first of the possibilities outlined above is correct, iron would be localized in intracellular stores (as hotspots) within the cytosol of infected, and of soon-to-be infected cells; in the second scenario, relatively high levels of iron in the epidermis of the nodule are observed; while in the third scenario, iron would be abundant around the vasculature.

Our studies indicate that iron is provided through the vasculature by the host plant, i.e. it is not the result of increased iron uptake at the nodule epidermal cells. Iron is released into the apoplast at zone II of the nodule, where from infected cells will absorb and transfer the metal to differentiating bacteroids. However, as the nodule senesces, the plant will recover at least some of this iron to be most likely used in flowering and embryo maturation.

\section{Experimental}

\section{Biological material and growth conditions}

Medicago truncatula A17 Jemalong seeds were scarified by incubating for $7 \mathrm{~min}$ with concentrated $\mathrm{H}_{2} \mathrm{SO}_{4}$. Washed, scarified seeds were surface sterilized with $50 \%$ bleach for $90 \mathrm{~s}$ and left overnight in sterile water. After $48 \mathrm{~h}$ at $4{ }^{\circ} \mathrm{C}$, seeds were germinated in water-agar plates at $22{ }^{\circ} \mathrm{C}$ for $48 \mathrm{~h}$. Seedlings were transferred to sterilized perlite pots and inoculated with Sinorhizobium meliloti 2011. Plants were grown in a greenhouse with $16 \mathrm{~h}$ of light and $22{ }^{\circ} \mathrm{C}$, and watered every two days with Jenner's solution ${ }^{17}$ or water, alternatively. Nodules were collected at 21 (mature) and 64 (senescent) dpi.

\section{Nodule fixation and embedding}

Nodules were fixed as in Punshon et al. (2011) with some modifications. ${ }^{18}$ Fixation buffer contained $4 \%$ formaldehyde, $0.25 \%$ glutaraldehyde, $2.5 \%$ sucrose in $50 \mathrm{mM}$ potassium phosphate buffer $\mathrm{pH}$ 7.4. Nodules were incubated overnight at $4{ }^{\circ} \mathrm{C}$ in this buffer with gentle agitation. Dehydration was carried out using an ethanol graded series $(0,30,50,70,85,90$, $95,100,100 \%$ in $2.5 \%$ sucrose, $50 \mathrm{mM}$ phosphate buffer $\mathrm{pH} 7.4$ ) at $4{ }^{\circ} \mathrm{C}$. Fixed and dehydrated nodules were infiltrated and embedded in LR-White (London Resin Company Ltd., UK) at $4{ }^{\circ} \mathrm{C}$. Embedded nodules were transferred to gelatin capsules and polymerized in fresh LR-White resin for $16 \mathrm{~h}$ at $60{ }^{\circ} \mathrm{C}$.

Sections were obtained using a Leica EM UC6 ultramicrotome. Semithin sections were stained with toluidine blue as reference for the S-XRF $5 \mu \mathrm{m}$ sections. The latter were transferred to $2 \times 2 \mathrm{~mm}$ silicon nitride windows (Silson, UK) for $\mathrm{S}$-XRF analyses.

\section{S-XRF data collection and analysis}

S-XRF data were collected at beamlines 8-BM-B and 2-ID-E at the Advanced Photon Source in Argonne National Laboratory (Argonne, IL). Incident X-rays were focused to a spot size of 5 or $0.5 \mu \mathrm{m}$ for 8-BM-B and 2-ID-E lines, respectively. Cells were raster-scanned in 5 (for 8-BM-B) or $0.5 \mu \mathrm{m}$ steps (for 2-ID-E), and fluorescence spectra were collected for either $2 \mathrm{~s}$ (8-BM-B) or $30 \mathrm{~ms}$ (2-ID-E) dwell times. Quantification and image processing were carried out using MAPS software. ${ }^{19}$ Standardization to convert a fluorescence signal to concentration $\left(\mu \mathrm{g} \mathrm{cm}^{-2}\right.$ ) was performed by fitting spectra to the signal from thin-film standards NBS-1832 and NBS-1833 (National Bureau of Standards, Gaithersburg, MD). To take into account biological variation, measurements were obtained from three different areas in each of 6 nodule sections, each derived from an individual nodule from a unique plant. A representative image for each developmental zone of the nodule is shown in Fig. 2-6.

\section{Results}

Low resolution S-XRF at beamline 8-BM-B was used to observe the general distribution of the elements in $M$. truncatula 

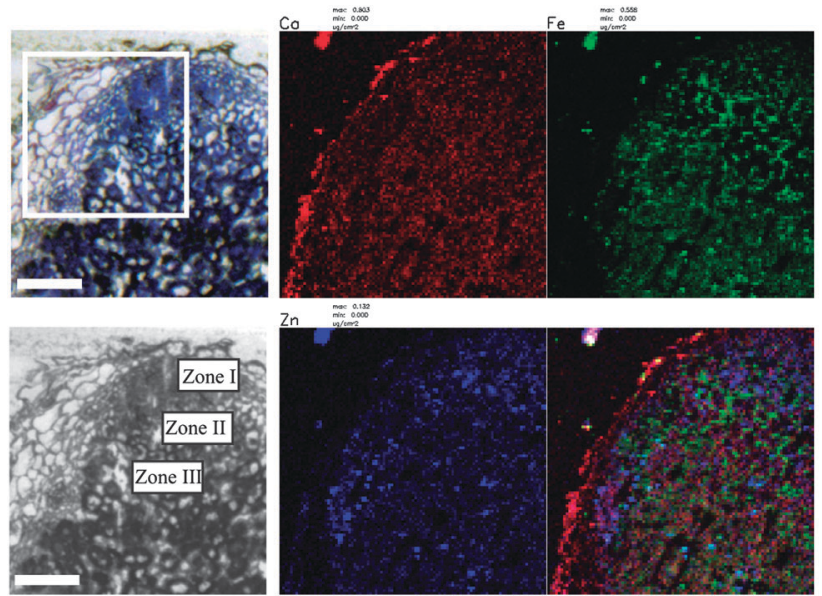

Fig. 2 Overview of Ca, Fe and Zn metal distribution in a M. truncatula nodule. Cross section of the nodule stained with toluidine blue (upper left panel) and the diagram of the different nodule regions (lower left panel). Scale bars indicate $100 \mu \mathrm{m}$. The boxed region was analysed by S-XRF at beamline 8-BM-B. Calcium is indicated in red, iron in green and zinc in blue (middle and right panels). Scale bar indicates $200 \mu \mathrm{m}$
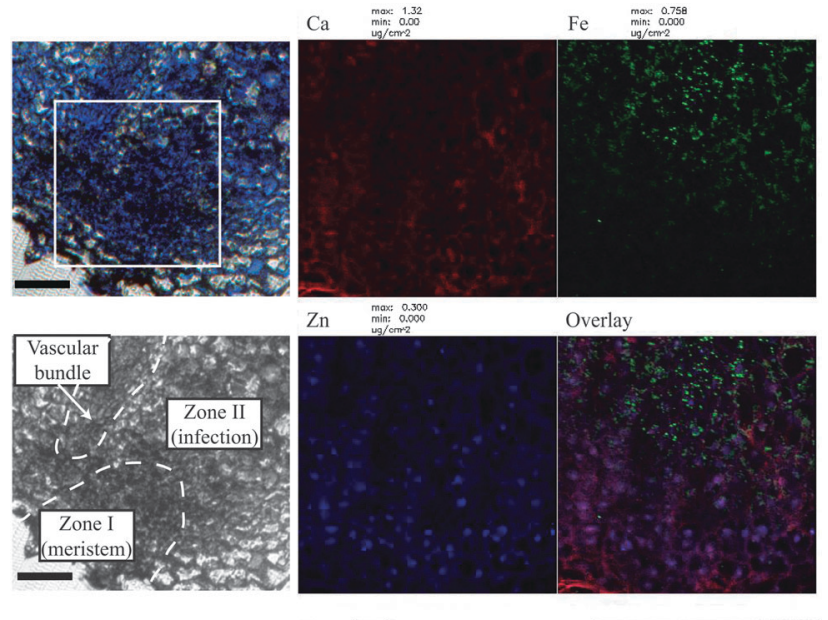

Fig. 3 Metal distribution in zone I and early zone II of the M. truncatula nodule Cross section of the nodule stained with toluidine blue (upper left panel) and the diagram of the different nodule regions (lower left panel). Scale bars indicate $50 \mu \mathrm{m}$. The boxed region was analysed by S-XRF at beamline 2-ID-E. Calcium is indicated in red, iron in green and zinc in blue (middle and right panels). Scale bar indicates $20 \mu \mathrm{m}$

nodules (Fig. 2 and Fig. S1, ESI $\dagger$ ). There was a defined localization of each element. Calcium was especially abundant at the interface between the cortical and the epidermal layer, where it could be reinforcing cell walls to minimize oxygen permeability. This element was also localized at high concentrations in bacteroidcontaining cells in zone III (Fig. 2). Potassium, in addition to having a similar distribution to calcium, was also predominantly localized in the meristematic, zone I, region (Fig. S1, ESI $\dagger$ ). Zinc was present as a number of hotspots, as well as distributed in a more or less circular pattern in the cortical cells (Fig. 2). Finally, iron was mostly confined to the proximities of the cortical cells,
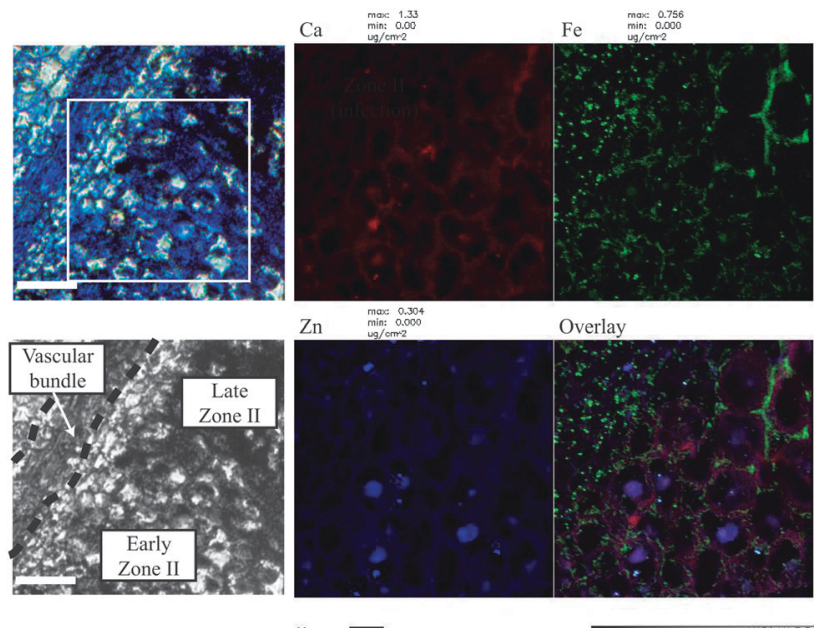

Fig. 4 Metal distribution in zone II of the M. truncatula nodule. Cross section of the nodule stained with toluidine blue (upper left panel) and the diagram of the different nodule regions (lower left panel). Scale bars indicate $50 \mu \mathrm{m}$. The boxed region was analysed by S-XRF at beamline 2-ID-E. Calcium is indicated in red, iron in green and zinc in blue (middle and right squares). Scale bar indicates $20 \mu \mathrm{m}$.
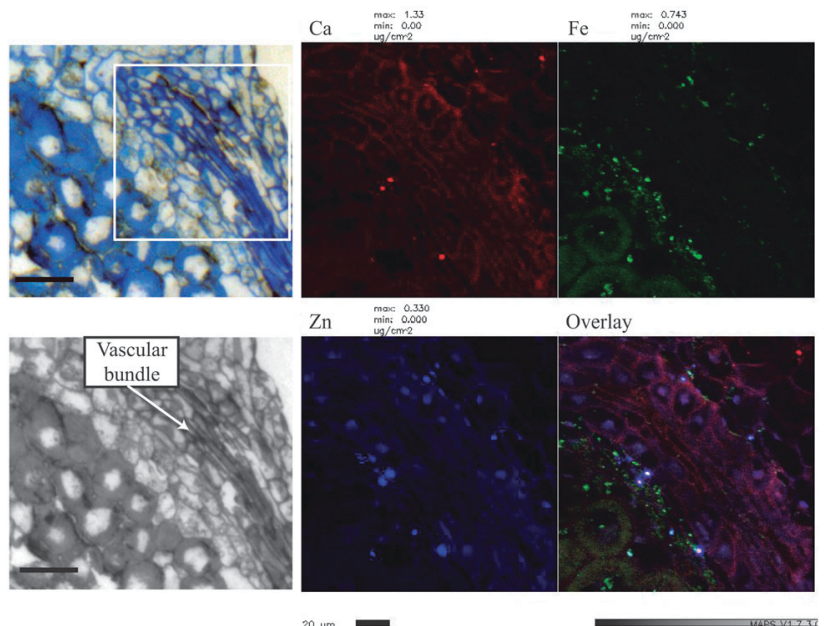

Fig. 5 Metal distribution in zone III of the $M$. truncatula nodule. Cross section of the nodule stained with toluidine blue (upper left panel) and the diagram of the different nodule regions (lower left panel). Scale bars indicate $50 \mu \mathrm{m}$. The boxed region was analysed by S-XRF at beamline 2-ID-E. Calcium is indicated in red, iron in green and zinc in blue (middle and right panels). Scale bar indicates $20 \mu \mathrm{m}$.

where it could be observed in zones II and III, having a more reticular distribution in zone II. Interestingly, very little iron was detected in zone I, and even less in the epidermal layers of the nodule (Fig. 2).

A closer look at higher resolution S-XRF indicated that zinc hotspots correspond to nuclei (Fig. 3). This is consistent with the abundance of zinc-finger domain regulatory proteins in the nucleus and with observations in other eukaryotic cell types. ${ }^{20}$ Smaller concentrations of this element were observed in the cell wall/apoplast regions of the cell, where calcium was the most abundant element. This is befitting the role of calcium in neutralizing and crosslinking negative charges, such as those of 


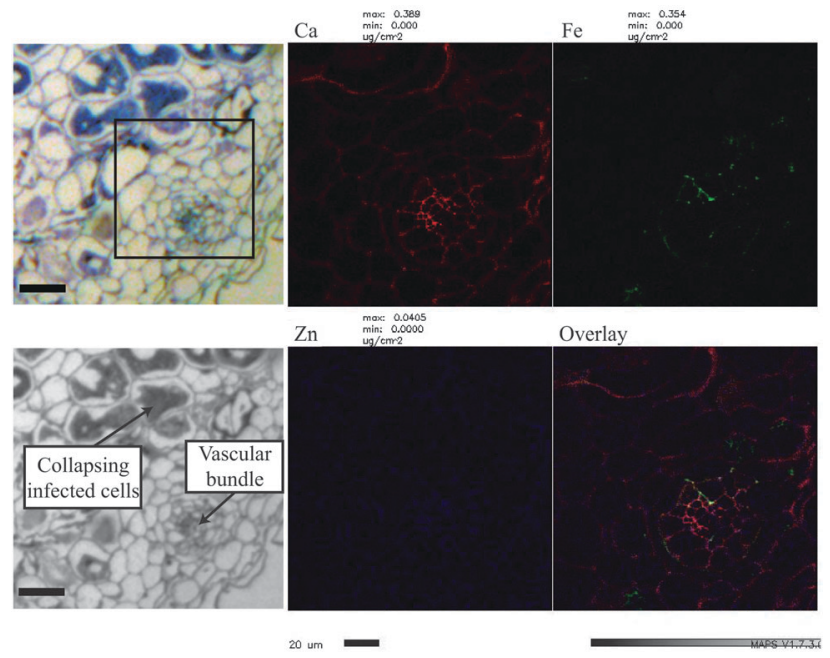

Fig. 6 Metal distribution in zone IV of the M. truncatula nodule. Cross section of the nodule stained with toluidine blue (upper left panel) and the diagram of the different nodule regions (lower left panel). Scale bars indicate $50 \mu \mathrm{m}$. The boxed region was analysed by S-XRF at beamline 2-ID-E. Calcium is indicated in red, iron in green and zinc in blue (middle and right panels). Scale bar indicates $20 \mu \mathrm{m}$.

pectins, in cell walls. Calcium was also observed in some nuclei (Fig. 3). As a result, zinc can be used as a nuclear marker and calcium as a cell wall marker, defining two additional compartments, the cytoplasm and the apoplasm, where these elements are present at much lower concentrations. This will enable the comparison of iron distribution in the different zones of the nodule.

As indicated in Fig. 2, iron was detected at very low concentrations in zone I, reaching much higher concentrations in early zone II. There, it did not have a continued distribution, but was present in a dotted pattern from the vascular bundle to the nodule cortical cells (Fig. 3). In some instances, a threadlike iron distribution was observed following the calcium-rich cell wall/apoplast. This iron distribution was maintained as zone II developed (Fig. 4). The iron-rich bodies appeared to be confined to the vasculature and its proximities, while the thread-like iron pattern developed between the cortical cells walls (indicated by calcium). As a result, iron accumulated in the apoplast at high levels. Iron hotspots were also observed within the zinc-rich nuclei (Fig. 2) corresponding to nucleoli. ${ }^{21}$ The distribution of calcium, and zinc remained unchanged even in older regions of zone II (Fig. 4).

Iron released into the apoplast of the zone II cortex was incorporated by the bacteroid-containing cells in zone III. The "doughnut"-like distribution of iron in these cells closely followed that of its bacteroids, with very little or no iron in the apoplast (Fig. 5). Nramp or ZIP transporters are most likely involved in the uptake of the apoplastic iron, since they are divalent cation importers ${ }^{13,22}$ with some of its members induced in nodules. ${ }^{23}$ This distribution pattern indicates that iron is released from the vasculature into the apoplast of zone II cells, in contrast to the two other possible pathways for iron delivery to the nodule. No metal accumulation was observed in the epidermal layer of the nodule, nor any iron-rich organelle appeared in the meristematic zone or in the infection/maturation zone. It is interesting to point out that iron is localized in small dense bodies very abundant in the vasculature. Given that iron is toxic when free, hydrated, in the cytosol, this observation would suggest some carrier system for iron delivery from the xylem to the apoplast. One possibility would be that iron is transported in vesicles and secreted to the apoplast. This system could, in theory, transport relatively large amounts of metals with a small energy investment while at the same time protecting the cytosol from Fenton-style reactions. In addition, the large accumulation of iron in a small area could explain the observed induction of ferritin in zones II and III, ${ }^{10 b, 24}$ since this protein is involved in iron accumulation and in detoxification of free-radicals produced in Fenton reactions. ${ }^{25}$

Some iron hotspots were also observed in non-nuclear regions of cells in early zone III (Fig. S2, ESI $\dagger$ ). They could correspond to intracellular membranes involved in the intense ferroprotein synthesis concomitant to nodulation. ${ }^{6,9 b}$ Alternatively, given the high levels of iron in the apoplast, an endocytic process might occur. This would allow for fast iron incorporation avoiding a damaging cytosolic iron accumulation. However, it would not explain the induction of Nramp and ZIP transporters in the nodule. ${ }^{32,33}$

One way or another, apoplastic iron clearly disappears in zone III. Part of it is incorporated into the symbiosomes, where it will be used to synthesize nitrogenase and other ferroproteins. This is a process necessarily mediated by transporters. Kaiser et al. (2003) proposed the involvement of Nramp transporter GmDMT1. ${ }^{12 b}$ However, biochemical evidence on Nramp transport suggests that its direction of transport is the opposite, towards, instead of from, the cytosol. ${ }^{13}$ A more likely candidate is SEN1, a VIT homologue whose mutation results in a nitrogen fixation deficient phenotype. ${ }^{14}$ The available evidence on the transport direction of VIT and its yeast homologue CCC1 indicates that they transport iron from cytosol to organelle, ${ }^{16 d, 26}$ which is compatible with a symbiosome iron loading function for SEN1.

In addition, iron was localized in the vascular region of zone III in globular shapes distinct from those present in the vasculature of zone II (Fig. 3 and 5). In these new structures, iron had a peripheral distribution and it was frequently associated with potassium-rich bodies (Fig. S3, ESI $\dagger$ ). Therefore, we might speculate that as iron has a different distribution in zone II and zone III vasculature, its direction of transport might be different. While zone II vascular iron vesicles would be involved in iron release to the apoplast, zone III vesicles could play a role in iron recycling back to the vasculature. Since this element is an essential limiting nutrient for plant growth, it would make sense that as little of it as possible remains "sequestered" in vesicles or in the apoplast, instead being recycled back to form new symbiosomes in zone II.

This metal distribution was altered upon senescence (Fig. 6). In zone IV, bacteroids collapsed from the cell periphery and their content was reused by the host plant. Calcium, zinc and iron concentrations were greatly diminished. The iron distribution pattern in the vasculature in zone IV was also different. 
Gone were the iron-rich hotspots or the K-rich bodies with the iron ring. In the senescence zone vasculature, iron was localized mostly within the vessel in a thread-like distribution. This is likely to be an important process required for recovering essential metals and translocating them back to the shoot. Since nodule senescence is coupled to flowering, ${ }^{27}$ it would be expected that nodular iron is recycled for flowering and embryogenesis as it is known to occur with iron from leaves. ${ }^{28}$ In fact, such a recycling occurs in zone IV of the nodule, where at least $50 \%$ of the iron is recovered. ${ }^{29}$ It has been shown that a nicotianamine synthase gene is induced in the senescent zone of the nodule. ${ }^{30}$ Since YSL transporters are involved in long-distance transport of iron-nicotianamine complexes, ${ }^{31}$ it seems probable that members of this family are involved in iron recycling from zone IV. In addition, ferritin would also play a role in accumulating iron in the vasculature, by controlling iron-loading of the phloem. ${ }^{10 b}$ The iron released in zone IV, could also be used as a nutrient by the saprophytic rhizobia living in close proximity. ${ }^{32}$

In our observations, we have not been able to obtain enough information to ascertain how other essential transition metals (zinc, copper, ...) are delivered to nodules. This is due to the much lower concentrations of these elements in the nodule. However, since most transition metals use similar transport mechanisms, ${ }^{33}$ it could be speculated that zinc, copper, manganese and similar elements could be transported in an iron-like fashion, i.e. being delivered by the vasculature and released into the apoplast of zone II. Nevertheless, available S-XRF data indicate that zinc plays an important role in the nucleus of nodular cells, as it occurs in other eukaryotes. $^{20}$ In addition, copper also colocalizes with zinc in meristematic and zone II cell nuclei (Fig. S4, ESI + ). This role of copper is novel and surprising, since no major copper containing protein has been associated with the nucleus, in part because the redox potential of copper would make it a dangerous element to have close to DNA.

Overall, S-XRF has been shown to be a suitable technique to study metal homeostasis in plant-microbe interactions, to our knowledge the first time that this is achieved. Data for metal homeostasis could potentially be affected by sample preparation. In this sense, methodologies proposed in this manuscript seem suitable, given that: (i) some of our observations have been reproduced by other methods, such as the nucleolar localization of iron, ${ }^{21}$ the zinc-rich nuclei, ${ }^{20}$ or the calcium-rich cell walls, ${ }^{34}$ (ii) iron distribution is not homogenous in the nodule, and (iii) ions were present in the locations where they were expected, such as iron in ferroprotein-rich bacteroids or potassium in the meristems (potassium has been linked to plant cell expansion and division ${ }^{35}$ ). Similar approaches could be used to study the role of metals in other plant, and in general host, interactions with microorganisms, such as pathogenesis. Given the potential of this technology and the critical role that metals play in the pathogenic process, ${ }^{36}$ new insights could be obtained on the role of metal partitioning in this interaction.

\section{Conclusions}

Legumes are among the most important crops in the world. Key to their success is their symbiosis with Rhizobium that enables

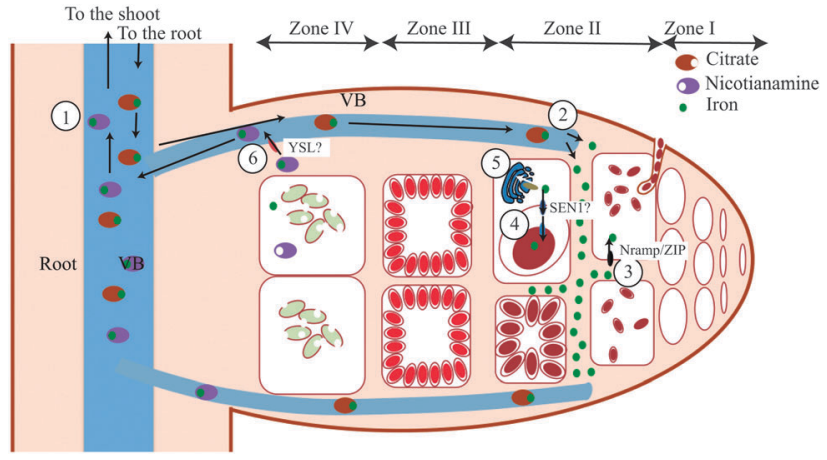

Fig. 7 Proposed model for iron transport in the nodule. Iron is transported associated to organic molecules through the vasculature $(V B, 1)$ and released into the apoplast of zone II (2). This process might be mediated by intracellular trafficking. Epidermal iron transporters of the infected cell introduce iron into the cytosol (3). Likely candidates are Nramp and ZIP family members. Once in the cytosol, iron can be transported into the symbiosomes, most likely through SEN1 or other VIT homologue (4). In addition, iron is translocated to other organelles for plant ferroprotein synthesis (5). Once the nodule senesces, iron is recycled and transferred back to the vasculature, probably through YSL transporters (6).

them to fix atmospheric nitrogen. Paramount to symbiosis is an appropriate supply of iron to the nodule for synthesis of all the key ferroproteins involved in the process. Using S-XRF we have shown that this metal reaches the maturation zone of the nodule through the vasculature, and it is released to the apoplast. From there, iron is taken up by the infected cells and delivered to symbiosomes. However, since iron is a scarce nutrient, upon flowering and the ensuing nodule senescence, it is recovered from the nodule and translocated to the shoot. In this work, S-XRF has proven to be an invaluable technique to trace elements in the different areas of the nodule. All these observations have allowed us to propose a model for iron transport in the nodule, summarized in Fig. 7. Future work will be directed towards identifying and characterizing the transporters mediating iron delivery and recovery.

\section{Acknowledgements}

This work was supported by the Ramón y Cajal Fellowship RYC2010-06363, and the Marie Curie International Reintegration Grant MENOMED (both to MGG). Use of the Advanced Photon Source was supported by the U.S. Department of Energy under contract number DE-AC02-06CH11357. We thank Dr Benoit Lefebvre (LIPM) for providing M. truncatula seeds, Drs Michael Udvardi and Igor Kryvoruchko (Noble Foundation) for critical reading of the manuscript and helpful discussion, and Drs José Rodrigo and Javier de Felipe (Centro de Tecnología Biomédica, UPM) for their help in preparing thin sections.

\section{References}

1 V. Smil, Nitrogen in crop production: an account of global flows, Global Biogeochem. Cycles, 1999, 13, 647-662.

2 P. van Rhijn and J. Vanderleyden, The Rhizobium-plant symbiosis, Microbiol. Rev., 1995, 59, 124-142. 
3 (a) E. Kondorosi, Z. Banfalvi and A. Kondorosi, Physical and genetic analysis of a symbiotic region of Rhizobium meliloti: identification of nodulation genes, Mol. Gen. Genet., 1984, 193, 445-452; (b) T. V. Bhuvaneswari and B. Solheim, Root hair deformation in the white clover/Rhizobium trifolii symbiosis, Physiol. Plant., 1985, 63, 25-31; (c) N. J. Brewin, Development of the legume root nodule, Annu. Rev. Cell Biol., 1991, 7, 191-226.

4 E. Limpens, S. Ivanov, W. van Esse, G. Voets, E. Fedorova and $\mathrm{T}$. Bisseling, Medicago $\mathrm{N}_{2}$-fixing symbiosomes acquire the endocytic identity marker Rab7 but delay the acquisition of vacuolar identity, Plant Cell, 2009, 21, 2811-2828.

5 J. Vasse, F. de Billy, S. Camut and G. Truchet, Correlation between ultrastructural differentiation of bacteroids and nitrogen fixation in alfalfa nodules, J. Bacteriol., 1990, 172, 4295-4306.

6 G. Ditta, E. Virts, A. Palomares and C. H. Kim, The nifA gene of Rhizobium meliloti is oxygen regulated, J. Bacteriol., 1987, 169, 3217-3223.

7 J. I. Sprent, Evolving ideas of legume evolution and diversity: a taxonomic perspective on the occurrence of nodulation, New Phytol., 2007, 174, 11-25.

8 (a) C. Tang, A. D. Robson and M. J. Dilworth, The role of iron in nodulation and nitrogen fixation in Lupinus angustifolius $\mathrm{L}$, New Phytol., 1990, 114, 173-182; (b) C. X. Tang, A. D. Robson, M. J. Dilworth and J. Kuo, Microscopic evidence on how irondeficiency limits nodule initiation in Lupinus angustifolius L, New Phytol., 1992, 121, 457-467.

9 (a) S. Moreau, J. M. Meyer and A. Puppo, Uptake of iron by symbiosomes and bacteroids from soybean nodules, FEBS Lett., 1995, 361, 225-228; (b) C. A. Appleby, Leghemoglobin and Rhizobium respiration, Annu. Rev. Plant Physiol., 1984, 35, 443-478.

10 (a) R. W. Miller, Z. Yu and C. G. Zarkadas, The nitrogenase proteins of Rhizobium meliloti: purification and properties of the MoFe and Fe components, Biochim. Biophys. Acta, 1993, 1163, 31-41; (b) P. M. Strozycki, A. Szczurek, B. Lotocka, M. Figlerowicz and A. B. Legocki, Ferritins and nodulation in Lupinus luteus: iron management in indeterminate type nodules, J. Exp. Bot., 2007, 58, 3145-3153.

11 R. E. Terry, K. U. Soerensen, V. D. Jolley and J. C. Brown, The role of active Bradyrhizobium japonicum in iron stress response of soy-beans, Plant Soil, 1991, 130, 225-230.

12 (a) S. Moreau, R. M. Thomson, B. N. Kaiser, B. Trevaskis, M. L. Guerinot, M. K. Udvardi, A. Puppo and B. A. Day, GmZIP1 encodes a symbiosis-specific zinc transporter in soybean, J. Biol. Chem., 2002, 277, 4738-4746; (b) B. N. Kaiser, S. Moreau, J. Castelli, R. Thomson, A. Lambert, S. Bogliolo, A. Puppo and D. A. Day, The soybean NRAMP homologue, GmDMT1, is a symbiotic divalent metal transporter capable of ferrous iron transport, Plant J., 2003, 35, 295-304.

13 Y. Nevo and N. Nelson, The NRAMP family of metal-ion transporters, Biochim. Biophys. Acta, 2006, 1763, 609-620.

14 T. Hakoyama, K. Niimi, T. Yamamoto, S. Isobe, S. Sato, Y. Nakamura, S. Tabata, H. Kumagai, Y. Umehara,
K. Brossuleit, T. R. Petersen, N. Sandal, J. Stougaard, M. K. Udvardi, M. Tamaoki, M. Kawaguchi, H. Kouchi and N. Suganuma, The integral membrane protein SEN1 is required for symbiotic nitrogen fixation in Lotus japonicus nodules, Plant Cell Physiol., 2012, 53, 225-236.

15 K. Takanashi, K. Yokosho, K. Saeki, A. Sugiyama, S. Sato, S. Tabata, J. F. Ma and K. Yazaki, LjMATE1: a citrate transporter responsible for iron supply to the nodule infection zone of Lotus japonicus, Plant Cell Physiol., 2013, 54, 585-594.

16 (a) L. Finney, S. Mandava, L. Ursos, W. Zhang, D. Rodi, S. Vogt, D. Legnini, J. Maser, F. Ikpatt, O. I. Olopade and D. Glesne, X-ray fluorescence microscopy reveals large-scale relocalization and extracellular translocation of cellular copper during angiogenesis, Proc. Natl. Acad. Sci. U. S. A., 2007, 104, 2247-2252; (b) S. Kehr, M. Malinouski, L. Finney, S. Vogt, V. M. Labunskyy, M. V. Kasaikina, B. A. Carlson, Y. Zhou, D. L. Hatfield and V. N. Gladyshev, X-ray fluorescence microscopy reveals the role of selenium in spermatogenesis, J. Mol. Biol., 2009, 389, 808-818; (c) T. Punshon, M. L. Guerinot and A. Lanzirotti, Using synchrotron X-ray fluorescence microprobes in the study of metal homeostasis in plants, Ann. Bot., 2009, 103, 665-672; (d) S. A. Kim, T. Punshon, A. Lanzirotti, L. Li, J. M. Alonso, J. R. Ecker, J. Kaplan and M. L. Guerinot, Localization of iron in Arabidopsis seed requires the vacuolar membrane transporter VIT1, Science, 2006, 314, 1295-1298.

17 P. Somasegaran and H. J. Hoben, Handbook for rhizobia: methods in legume-Rhizobium technology, Springer, New York, 1994.

18 T. Punshon, K. Hirschi, J. Yang, A. Lanzirotti, B. Lai and M. L. Guerinot, The role of CAX1 and CAX3 in elemental distribution and abundance in Arabidopsis seed, Plant Physiol., 2011, 158, 352-362.

19 S. Vogt, MAPS: a set of software tools for analysis and visualization of 3D X-ray fluorescence data sets, J. Phys. IV, 2003, 104, 635-638.

20 J. L. Wolford, Y. Chishti, Q. Jin, J. Ward, L. Chen, S. Vogt and L. Finney, Loss of pluripotency in human embryonic stem cells directly correlates with an increase in nuclear zinc, PLoS One, 2010, 5, e12308.

21 H. Roschzttardtz, L. Grillet, M. P. Isaure, G. Conejero, R. Ortega, C. Curie and S. Mari, Plant cell nucleolus as a hot spot for iron, J. Biol. Chem., 2011, 286, 27863-27866.

22 D. Eide, The ABC of solute carriers. The SLC39 family of metal ion transporters, Eur. J. Physiol., 2004, 447, 796-800.

23 (a) D. S. Lohar, N. Sharopova, G. Endre, S. Peñuela, D. Samac, C. D. Town, K. A. T. Silverstein and K. A. VandenBosch, Transcript analysis of early nodulation events in Medicago truncatula, Plant Physiol., 2006, 140, 221-234; (b) V. A. Benedito, I. Torres-Jerez, J. D. Murray, A. Andriankaja, S. Allen, K. Kakar, M. Wandrey, J. Verdier, H. Zuber, T. Ott, S. Moreau, A. Niebel, T. Frickey, G. Weiller, J. He, X. Dai, P. X. Zhao, Y. Tang and M. K. Udvardi, A gene expression atlas of the model legume Medicago truncatula, Plant J., 2008, 55, 504-513. 
24 M. M. Lucas, G. Van de Sype, D. Hérouart, M. J. Hernández, A. Puppo and M. R. de Felipe, Immunolocalization of ferritin in determinate and indeterminate legume root nodules, Protoplasma, 1998, 204, 61-70.

25 J. F. Briat, C. Duc, K. Ravet and F. Gaymard, Ferritins and iron storage in plants, Biochim. Biophys. Acta, 2010, 1800, 806-814.

26 L. Li, O. S. Chen, D. McVey Ward and J. Kaplan, CCC1 is a transporter that mediates vacuolar iron storage in yeast, J. Biol. Chem., 2001, 276, 29515-29519.

27 G. J. Bethlenfalvay and D. A. Phillips, Ontogenetic interactions between photosynthesis and symbiotic nitrogen fixation in legumes, Plant Physiol., 1977, 60, 419-421.

28 R. Shi, G. Weber, J. Köster, M. Reza-Hajiresaei, C. Zou, F. Zhang and N. von Wirén, Senescence-induced iron mobilization in source leaves of barley (Hordeum vulgare) plants, New Phytol., 2012, 195, 372-383.

29 J. W. Burton, C. Harlow and E. C. Theil, Evidence for reutilization of nodule iron in soybean seed development, J. Plant Nutr., 1998, 5, 913-927.

30 T. Hakoyama, H. Watanabe, J. Tomita, A. Yamamoto, S. Sato, Y. Mori, H. Kouchi and N. Suganuma, Nicotianamine synthase specifically expressed in root nodules of Lotus japonicus, Planta, 2009, 230, 309-317.

31 S. S. Conte and E. L. Walker, Transporter contributing to iron trafficking in plants, Mol. Plant, 2011, 4, 464-476.

32 A. C. J. Timmers, E. Soupène, M.-C. Auriac, F. de Billy, J. Vasse, P. Boistard and G. Truchet, Saprophytic intracellular rhizobia in alfalfa nodules, Mol. Plant-Microbe Interact., 2000, 13, 1204-1213.

33 (a) C. Curie, G. Cassin, D. Couch, F. Divol, K. Higuchi, M. Le Jean, J. Misson, A. Schikora, P. Czernic and S. Mari, Metal movement within the plant: contribution of nicotianamine and yellow stripe 1-like transporters, Ann. Bot., 2009, 103, 1-11; (b) N. Grotz and M. L. Guerinot, Molecular aspects of $\mathrm{Cu}, \mathrm{Fe}$ and $\mathrm{Zn}$ homeostasis in plants, Biochim. Biophys. Acta, 2006, 1763, 595-608; (c) S. Puig and L. Peñarrubia, Placing metal micronutrients in context: transport and distribution in plants, Curr. Opin. Plant Biol., 2009, 12, 299-306.

34 P. J. White and M. R. Broadley, Calcium in plants, Ann. Bot., 2003, 92, 487-511.

35 T. Sano, N. Kutsuna, D. Becker, R. Hedrich and S. Hasezawa, Outward-rectifying $\mathrm{K}+$ channel activities regulate cell elongation and cell division of tobacco BY-2 cells, Plant J., 2009, 57, 55-64. 36 (a) M. González-Guerrero, D. Raimunda, X. Cheng and J. M. Argüello, Distinct functional roles of homologous $\mathrm{Cu}+$ efflux ATPases in Pseudomonas aeruginosa, Mol. Microbiol., 2010, 78, 1246-1258; (b) D. Expert, Withholding and exchanging iron: interactions between Erwinia spp. and their plant hosts, Annu. Rev. Phytopathol., 1999, 37, 307-334; (c) T. E. Kehl-Fie and E. P. Skaar, Nutritional immunity beyond iron: a role for manganese and zinc, Curr. Opin. Chem. Biol., 2010, 14, 218-224; (d) J. M. Argüello, M. González-Guerrero and D. Raimunda, Bacterial transition metal P1B-ATPases: transport mechanism and roles in virulence, Biochemistry, 2011, 50, 9940-9949. 